\title{
TIME SERIES ANALYSIS OF SURFACE DEFORMATION OF BENGALURU CITY USING SENTINEL-1 IMAGES
}

\author{
Nikitha Ittycheria ${ }^{1}$, Divya Sekhar Vaka ${ }^{2}$, Y. S. Rao ${ }^{2}$ \\ ${ }^{1}$ Department of Applied Mechanics, National Institute of Technology Karnataka 575025, India - nikithaitty@gmail.com \\ ${ }^{2}$ Centre of Studies in Resources Engineering, Indian Institute of Technology Bombay, Mumbai 400076, India \\ - sekharv@iitb.ac.in, ysrao@csre.iitb.ac.in
}

Commission V, SS: Disaster Monitoring, Damage Assessment and Risk Reduction

KEYWORDS: Synthetic Aperture Radar, Persistent Scatterer Interferometry, Urban subsidence, Polarization

\begin{abstract}
:
Persistent Scatterer Interferometry (PSI) is an advanced technique to map ground surface displacements of an area over a period. The technique can measure deformation with a millimeter-level accuracy. It overcomes the limitations of Differential Synthetic Aperture Radar Interferometry (DInSAR) such as geometric, temporal decorrelation and atmospheric variations between master and slave images. In our study, Sentinel-1A Interferometric Wide Swath (IW) mode descending pass images from May 2016 to December 2017 (23 images) are used to identify the stable targets called persistent scatterers (PS) over Bengaluru city. Twenty-two differential interferograms are generated after topographic phase removal using the SRTM 30 m DEM. The main objective of this study is to analyze urban subsidence in Bengaluru city in India using the multi-temporal interferometric technique such as PSI. The pixels with Amplitude Stability Index $\geq 0.8$ are selected as initial PS candidates (PSC). Later, the PSCs having temporal coherence $>0.5$ are selected for the time series analysis. The number of PSCs that are identified after final selection are reduced from 59590 to 54474 for VV polarization data and 15611 to 15596 for $\mathrm{VH}$ polarization data. It is interesting to note that a very less number of PSC are identified in cross-polarized images (VH). A high number of PSC have identified in co-polarized (VV) images as the vertically oriented urban targets produce a double bounce, which results in a strong return towards the sensor. The velocity maps obtained using VV and VH polarizations show displacement in the range of $\pm 20 \mathrm{~mm} \mathrm{year}^{-1}$. The subsidence and the upliftment observed in the city shows a linear trend with time. It is observed that the eastern part of Bengaluru city shows more subsidence than the western part.
\end{abstract}

\section{INTRODUCTION}

Differential Interferometric Synthetic Aperture Radar (InSAR) is an efficient remote sensing tool for surface deformation mapping. DInSAR is widely used in the fields of land subsidence mapping, earthquake and volcanic deformation studies, landslide mapping, and glacier movements (Massonnet and Feigl, 1998). Persistent Scatterer Interferometry (PSI) is an advanced DInSAR technique, which exploits multiple images of interferometric synthetic aperture radar (SAR) data to estimate deformation over an area. It overcomes the limitations of the DInSAR technique such as geometric, temporal decorrelation and atmospheric variations between images (Hanssen, 2001). The use of time series of images brings out even smaller changes occurred in the Earth's surface in millimeter precision. The technique involves the process of master image selection, coregistration with respect to a single master image, differential interferogram generation, filtering, phase unwrapping, selection of persistent scatterers, atmospheric phase removal, time series analysis and displacement estimation of selected PSC. The key point of this technique is the identification of stable targets called 'persistent scatterer candidates (PSC).' The PSC are stable pixels that are present in all the selected images with the same scattering properties over time. It can be human-made objects like buildings, pipelines, electric poles or artificially installed corner reflectors. Generally, PSC can be detected very easily in urban environments because of the high-rise buildings. It is difficult to detect PSC in the rural areas due to the presence of agricultural fields or the vegetated areas that with time (Hooper et al., 2004).

The critical step in the PSI technique is the identification of stable targets. There are many methods to analyze the PSCs'. A statistical parameter called amplitude dispersion $\left(D_{A}\right)$ was used as PS the selection criterion in the PSInSAR approach proposed by Ferretti et al., $(2001,2000) . D_{A}$ is the ratio of standard deviation $\left(\sigma_{A}\right)$ and mean $\left(\mu_{A}\right)$ of a pixel in the temporal domain.

$$
D_{A}=\frac{\sigma_{A}}{\mu_{A}}
$$

For PS point selection, $D_{A}$ will range from 0 to 0.25 . To include distributed scatters, a value from 0 to 0.4 is considered. This algorithm was successful in analysing the PS points over urban areas but it fails to identify the PS points in rural areas. Werner et al., (2003) proposed an Interferometric Point Target Analysis (IPTA) method, which uses temporal variability and spectral phase diversity for PS selection. Hooper et al., (2007, 2004) proposed Stanford Method for Persistent Scatterers (StaMPS), which uses amplitude dispersion $\left(D_{A}\right)$ and a phase stability criterion to select and refine PS points. Permanent scatterers (PS) and distributed scatterers (DS) are identified using this technique.

Most of the deformation estimation studies depending on the data availability used either $\mathrm{HH}$ polarization (Wegmuller et al., 2010) or VV polarization ( $\mathrm{Ng}$ et al., 2012) images to study time series deformation over an area. The number of PS points identified using the PSI technique varies depending on the polarization used for time series estimation. Even though the line-of-sight deformation magnitude is similar, the number of PSC identified in VV are much higher than that of $\mathrm{HH}$ polarization for urban areas (Vaka et al., 2017).

In this study, $\mathrm{VV}$ and $\mathrm{VH}$ polarization single-look-complex (SLC) images of Sentinel-1A satellite are used to study the difference in the number of PSC and the difference in the deformation estimates over Bengaluru city in India using the multi-temporal interferometric technique such as PSI. 


\section{STUDY AREA AND DATASETS}

\subsection{Study Area}

The Bengaluru city is situated within the latitude and longitude of $12^{\circ} 45^{\prime} \mathrm{N}-13^{\circ} 10^{\prime} \mathrm{N}$ and $77^{\circ} 25^{\prime} \mathrm{E}-77^{\circ} 45^{\prime} \mathrm{E}$, and accounts an area of 720 sq.km (see Figure 1). Bengaluru called the megacity is the third most populated city in India. It is the capital of Karnataka state, India. It lies in an elevation over $900 \mathrm{~m}$ above mean sea level. Bangalore often referred as 'IT Capital of India' accounts for a population of about 8 million. The city has a moderate climate throughout the year with an average low temperature of $15.1^{\circ} \mathrm{C}$ and a high temperature of $35^{\circ} \mathrm{C}$. The population growth between 2001 and 2011 has been increasing by more than $300 \%$ in the outer areas (Sekhar et al., 2017).
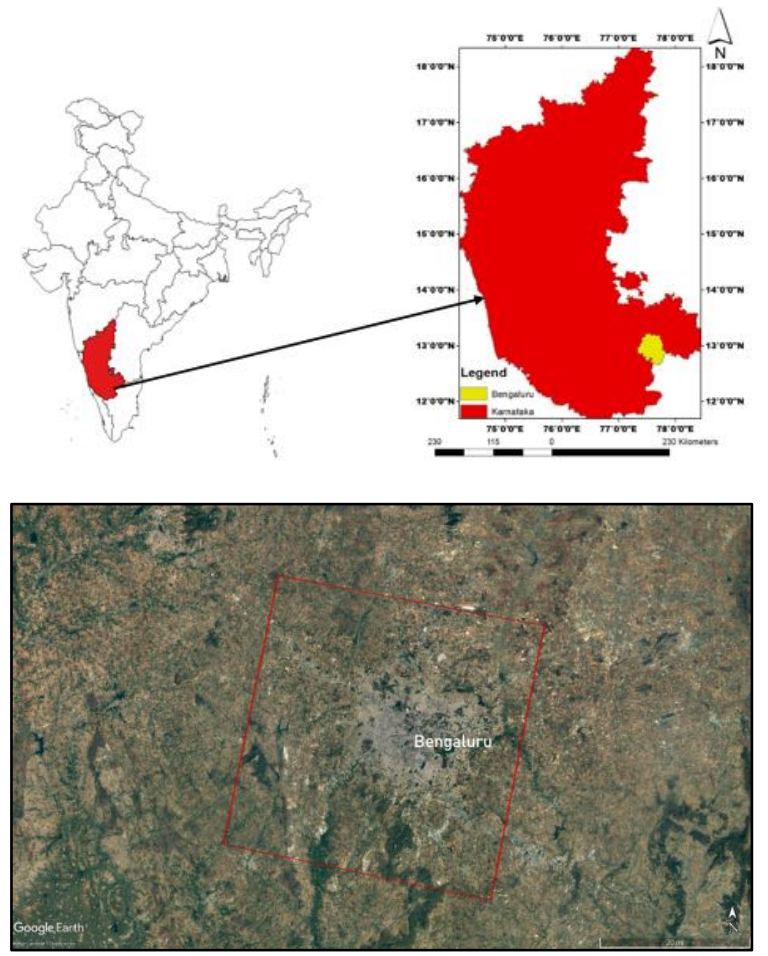

Figure 1: Bengaluru study area. The red square over Google Earth imagery represents the 25 sq. km. area considered for the present study.

\subsection{Datasets}

In the present study, we have used twenty-three Sentinel-1A descending pass images from 15-05-2016 to 24-12-2017 to study the ground surface deformation pattern of Bengaluru city. The Sentinel-1A data used in this study are acquired in Interferometric Wide Swath (IW) mode with a spatial resolution of $5 \mathrm{~m} \times 20 \mathrm{~m}$ in range and azimuth direction respectively. In this mode, three subswaths are captured using Terrain Observation with Progressive Scans (TOPS) technique and each subswath contain series of bursts (De Zan and Guarnieri, 2006). The data is made accessible to the users through the Copernicus Open Access Hub. A 25 sq.km. area covering the city is chosen for the time series analysis. Both VV and VH polarization images are considered separately for time series analysis. An image acquired on 14-08-2017 is selected as the master image in both polarizations. The perpendicular baseline between master and slave images are plotted as an images graph in Figure 2. The incidence angle at mid swath is $38.3^{\circ}$. The maximum normal baseline between a pair of images is $100 \mathrm{~m}$.

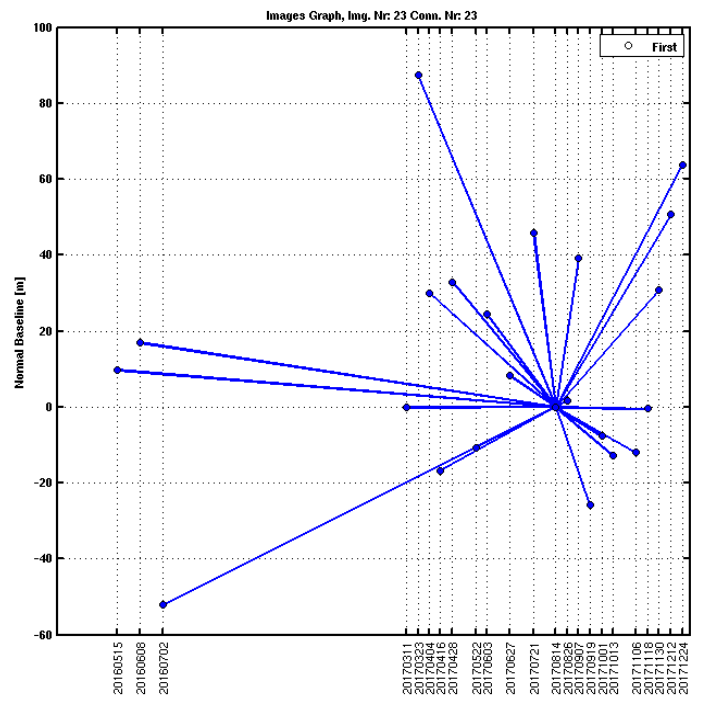

Figure 2: Images graph showing connections between Sentinel-1 images. The $\mathrm{x}$-axis represents the date of acquisition and the $\mathrm{y}$ axis represents the normal/perpendicular baseline in meters. Each link represents an interferogram. Twenty-two interferograms are formed using 23 images between May 2016 and December 2017.

\section{METHODOLOGY}

The master and slave images are extracted and co-registered with a single master image, which is acquired on 14-08-2017. After co-registration of 23 Sentinel-1A images, twenty-two interferograms are formed. An SRTM $30 \mathrm{~m}$ DEM is used for topographic phase removal. The DEM is converted into radar coordinates and subtracted from interferograms to generate differential interferograms. These differential interferograms are then Goldstein phase filtered before giving them as an input for time series analysis. Amplitude images are calculated from SLCs and PS points are selected based on an amplitude-based criterion. The pixels with Amplitude Stability Index (ASI) $\geq 0.8$ are selected as initial PS candidates (PSC). This criterion is equivalent to Amplitude Dispersion $\left(D_{A}\right)<0.2$. The relation between ASI and $D_{A}$ is given in Eq.2. Later, the PSCs having temporal coherence $>0.5$ are selected for the time series analysis. Among all the PS points, one stable target, which remains constant over time, was selected as a reference point. Figure 3 represents the methodology followed for surface deformation estimation of Bengaluru city.

$$
A S I=1-D_{A}=1-\frac{\sigma_{A}}{\mu_{A}}
$$

In the process of time series estimation, the DEM error and atmospheric phase errors are also estimated and removed. The error in DEM may introduce residual errors in height estimation, which is termed as Residual Topographic Error (RTE) or simply DEM error. The estimated values of residual topographic error are subtracted from differential interferograms by converting height error (from meters) into corresponding phase values (to radian). The phase delays that are introduced due to the atmospheric variations between the master and slave acquisitions are a significant problem in deformation monitoring using PSI technique. As a result of the spatial or temporal difference in relative humidity, the atmospheric phase delays can generate 10$14 \mathrm{~cm}$ errors in deformation measurements (Zebker et al., 1997). Therefore, the effects due to atmospheric phase should be 
removed. High pass filtering in the time domain and low pass filtering in the spatial domain are performed to correct for atmospheric phase delays. Also, temperature, pressure, humidity and rainfall data of the study area are also used for atmospheric corrections. After atmospheric phase removal, displacement and velocity map of the Bengaluru area are estimated. The estimated deformation phase of each PSC is converted into range change measurements. Velocity maps showing deformation per year for both VV and VH polarizations are generated.

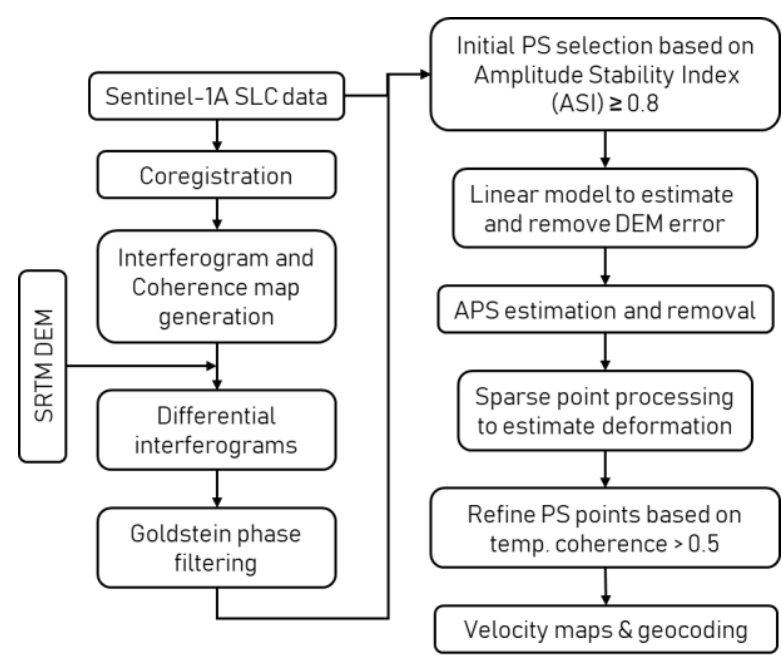

Figure 3: Methodology followed for surface deformation estimation of Bengaluru city.

\section{RESULTS AND DISCUSSIONS}

Using Sentinel-1A SLC data, subsidence analysis of Bengaluru is done using $\mathrm{VV}$ and $\mathrm{VH}$ polarizations. Time series analysis of 22 interferograms showed noticeable deformation. Number of PSC obtained initially in VV and VH polarizations are 59590 and 15611. After refinement (i.e., Amplitude stability index $\geq 0.8$ and temporal coherence > 0.5), the number of PSC reduced to 54474 in VV polarization and 15596 in $\mathrm{VH}$ polarization. It is interesting to note that a very less amount of PSC are identified in crosspolarized images $(\mathrm{VH})$. A high number of PSC have identified in co-polarized (VV) images as the vertically oriented urban targets produce a double bounce, which results in a substantial return towards the sensor.

In both VV and VH polarizations subsidence in the eastern part and upliftment in the western part of the city are observed. The observed subsidizing areas lie in the outskirts of the town in the areas of Electronic City, Anekkal, Kalyan Nagar, etc. The deformation trend shows a linear pattern, which ranges from 10 to $20 \mathrm{~mm}$. Figure 4 shows the resampled velocity maps obtained after time series analysis of $\mathrm{VV}$ and $\mathrm{VH}$ polarizations images. The deformation trend with respect to time at few subsidence and upliftment points are shown in Figure 5.

From the analysis, it is clear that an eastern part of Bengaluru city is subsidizing at an average rate of $15 \mathrm{~mm}_{\text {year-1 }}^{-1}$ to $20 \mathrm{~mm}_{\text {year }}{ }^{-1}$. Both humanmade and natural activities lead to the gradual subsidence in most of the cities. The Bengaluru city depends mainly on Cauvery River water and groundwater to meet their daily water requirements. Even though the availability of surface water has increased, it failed to catch up with the anthropogenic growth and as a result, groundwater is being extracted in the city to meet the day-to-day needs. This led to the digging of more borewells in the city. In various sectors like domestic, industrial or government organizations, the groundwater use is not according to regulations. Thus, the groundwater extraction without artificial recharge tends to be one of the main reason causing subsidence. However, in urban areas, the groundwater recharge is hindered by the influence of built-up, impervious regions (Sekhar et al., 2017). Consolidation may occur in clay land or soil with low permeability. The occurrence of irreversible shrinkage after an improvement in the drainage conditions leads to consolidation. Another major problem of Bengaluru city is that the groundwater penetration is very low and most of the water will flow as surface runoff. As the city is growing at a very high pace, the construction of buildings over the swamp areas also can cause subsidence. In a study conducted by the Centre for Ecological Sciences at the Indian Institute of Science, Bengaluru it is found to be a 525\% growth in built-up areas, $70 \%$ decline in the number of water bodies, and rapid decline in vegetation.

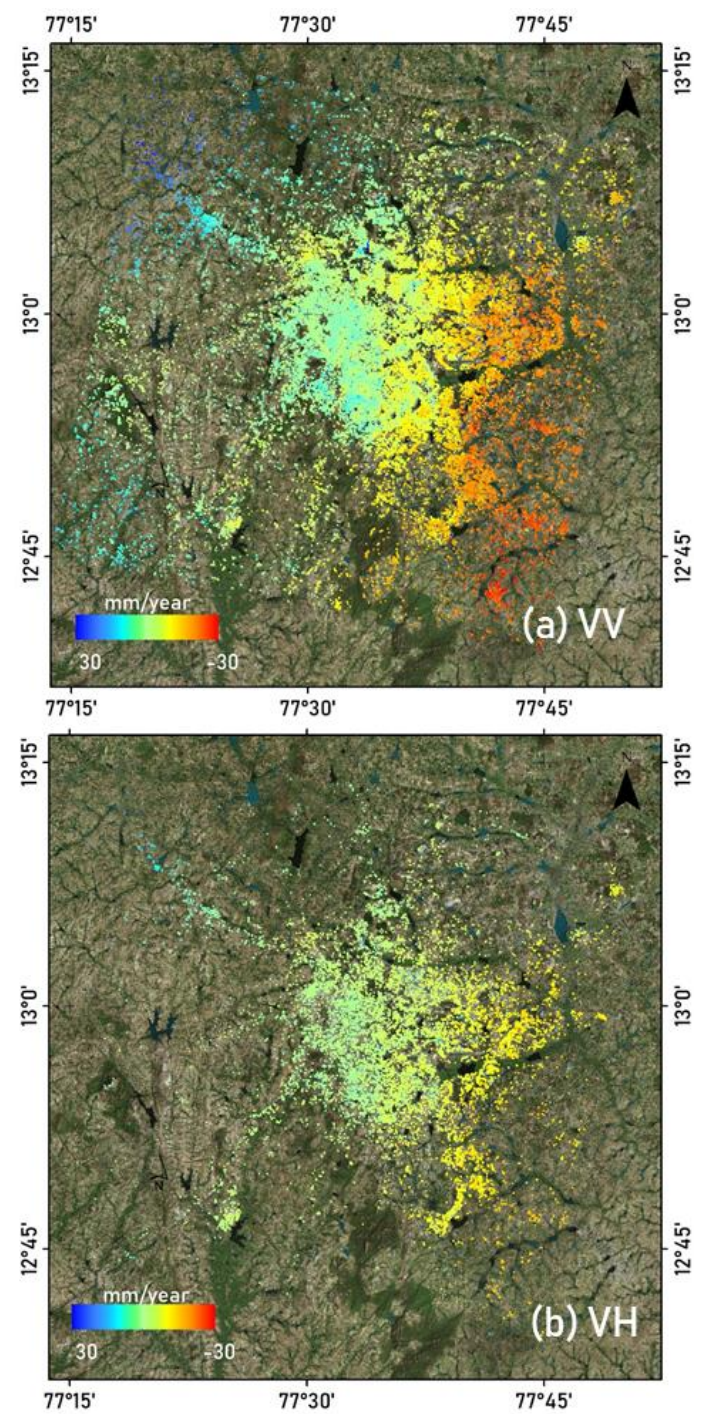

Figure 4: Resampled velocity maps of (a) VV and (b) VH polarizations over Bengaluru city. A high deformation is observed in the co-polarization (VV) velocity map due to its response to urban settlements. Maximum and minimum deformation rates are shown in $\mathrm{mm} / \mathrm{year}$. The eastern part of Bengaluru city shows more subsidence than the western part. 
Due to the improper drainage, which leads to water flow over the surface thus reducing the soil bearing capacity and construction of buildings over these can lead to subsidence in the nearby areas. In some areas, the construction of buildings is done without proper compaction and filling. The presence of nearby lakes in the region may also add up to the cause of subsidence. Natural phenomenon like tectonic movements, the subsurface solution of rock salt, gypsum, or carbonate rocks, can all cause subsidence. The present study does not show much upliftment. The observed upliftment pattern may be due to the ongoing construction process.

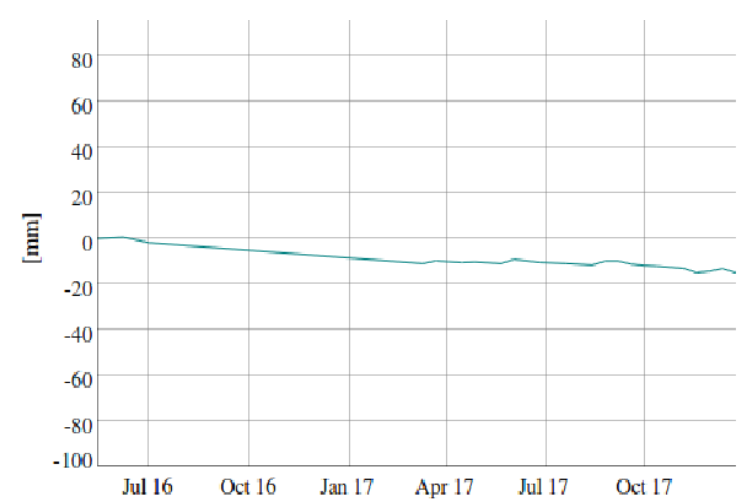

a) Subsidence point in VV polarization at $77.67^{\circ} \mathrm{E}, 12.88^{\circ} \mathrm{N}$.

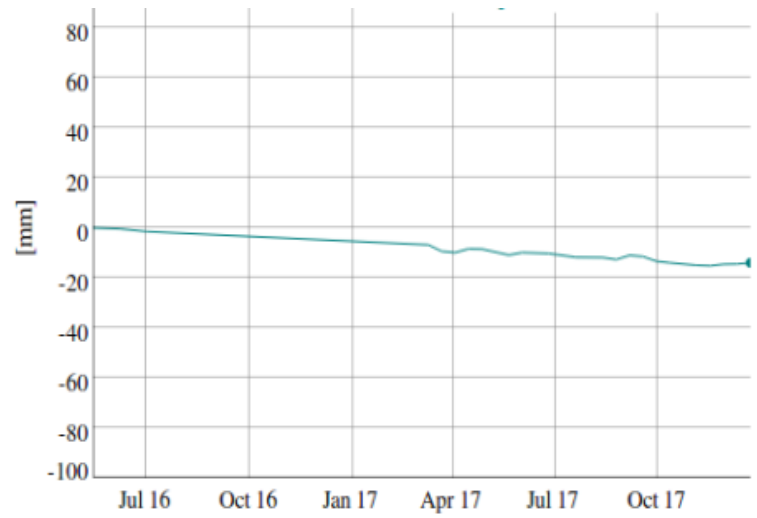

b) Subsidence point in $\mathrm{VH}$ polarization at $77.66^{\circ} \mathrm{E}, 12.86^{\circ} \mathrm{N}$.

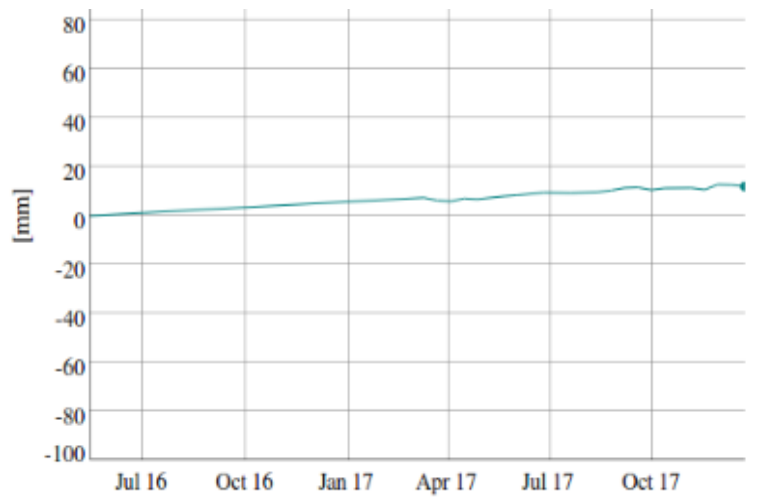

c) Uplift point in $\mathrm{VV}$ polarization at $77.31^{\circ} \mathrm{E}, 12.79^{\circ} \mathrm{N}$.

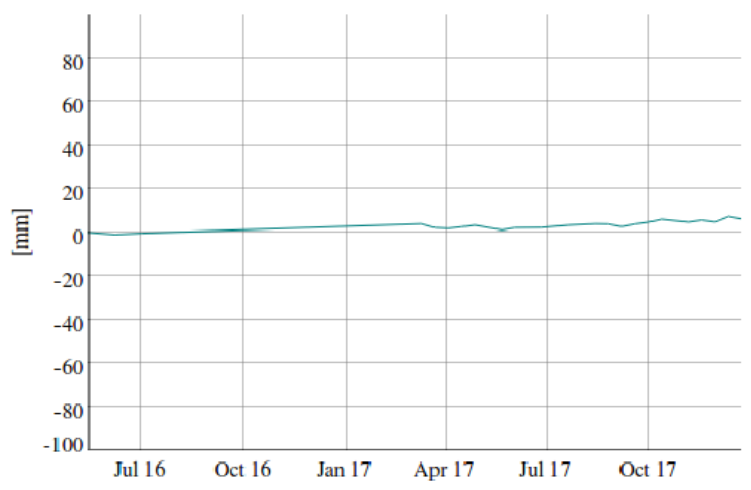

d) Uplift point in $\mathrm{VH}$ polarization at $77.57^{\circ} \mathrm{E}, 13.05^{\circ} \mathrm{N}$.

Figure 5: Graphs showing deformation trend at subsidence upliftment points in $\mathrm{VV}$ and $\mathrm{VH}$ polarizations.

\section{CONCLUSION}

The Sentinel 1A data are analyzed to study the land subsidence in Bengaluru city. The effect of VV and VH polarizations on the deformation estimates is studied. The PSC identified using VV polarization are more than that of using $\mathrm{VH}$ polarization as the vertically oriented urban targets produce a double bounce, which results in a substantial return towards the sensor. Both polarizations show nearly similar deformation pattern. Eastern part of the city area shows subsidence. This may be due to humanmade and natural activities. From May 2016 to December 2017, the deformation follows the assumed linear deformation model. Our results depict the difference in identification of PSC using VV and VH polarizations. The future study can include the ground truth data obtained using GPS and leveling methods at various points within the city and can be verified with the SAR predictions.

\section{ACKNOWLEDGMENTS}

The Sentinel-1 data are provided free of cost by European Space Agency (ESA) through the Copernicus Open Access Hub. We are grateful to SARPROZ team for giving an evaluation copy of the software tool.

\section{REFERENCES}

De Zan, F., Guarnieri, A.M., 2006. TOPSAR: Terrain observation by progressive scans. IEEE Transactions on Geoscience and Remote Sensing 44, 2352-2360. https://doi.org/10.1109/TGRS.2006.873853

Ferretti, A., Prati, C., Rocca, F., 2001. Permanent scatterers in SAR interferometry. IEEE Transactions on geoscience and remote sensing 39, 8-20.

https://doi.org/10.1109/IGARSS.1999.772008

Ferretti, A., Prati, C., Rocca, F., 2000. Nonlinear subsidence rate estimation using permanent scatterers in differential SAR interferometry. IEEE Transactions on Geoscience and Remote Sensing 38, 2202-2212. https://doi.org/10.1109/36.868878

Hanssen, R.F., 2001. Radar interferometry: data interpretation and error analysis. Springer Science \& Business Media.

Hooper, A., Segall, P., Zebker, H., 2007. Persistent scatterer interferometric synthetic aperture radar for crustal deformation 
analysis, with application to Volcán Alcedo, Galápagos. Journal of Geophysical Research: Solid Earth 112

https://doi.org/10.1029/2006JB004763

Hooper, A., Zebker, H., Segall, P., Kampes, B., 2004. A new method for measuring deformation on volcanoes and other natural terrains using InSAR persistent scatterers. Geophysical research letters 31 .

https://doi.org/10.1029/2004GL021737

Massonnet, D., Feigl, K.L., 1998. Radar interferometry and its application to changes in the Earth's surface. Reviews of geophysics 36, 441-500.

https://doi.org/10.1029/97RG03139

Ng, A.H.-M., Ge, L., Li, X., Zhang, K., 2012. Monitoring ground deformation in Beijing, China with persistent scatterer SAR interferometry. Journal of Geodesy 86, 375-392. https://doi.org/10.1007/s00190-011-0525-4

Sekhar, M., Tomer, S.K., Thiyaku, S., Giriraj, P., Murthy, S., Mehta, V.K., 2017. Groundwater Level Dynamics in Bengaluru City, India. Sustainability 10, 26.
Vaka, D.S., Sharma, S., Rao, Y.S., 2017. Comparison of HH and VV Polarizations for Deformation Estimation using Persistent Scatterer Interferometry, in: 38th Asian Conference on Remote Sensing - Space Applications: Touching Human Lives, ACRS 2017. AARS, New Delhi, p. unpaginated.

Wegmuller, U., Walter, D., Spreckels, V., Werner, C.L., 2010. Nonuniform ground motion monitoring with TerraSAR-X persistent scatterer interferometry. IEEE Transactions on Geoscience and Remote Sensing 48, 895-904. https://doi.org/10.1109/TGRS.2009.2030792

Werner, C., Wegmuller, U., Strozzi, T., Wiesmann, A., 2003. Interferometric point target analysis for deformation mapping, in: Geoscience and Remote Sensing Symposium, 2003. IGARSS'03. Proceedings. 2003 IEEE International. IEEE, pp. 4362-4364. https://doi.org/10.1109/IGARSS.2003.1295516

Zebker, H.A., Rosen, P.A., Hensley, S., 1997. Atmospheric effects in interferometric synthetic aperture radar surface deformation and topographic maps. Journal of Geophysical Research: $\quad$ Solid Earth 102, 7547-7563. https://doi.org/10.1029/96JB03804 